\title{
The Viewpoint from Commission 40
}

\author{
Richard N. Manchester \\ CSIRO, Australian National Telescope Facility, Sydney, Australia
}

\begin{abstract}
.
Although Very Long Baseline Interferometry has resolved a few binary stellar systems, pulsars provide the main source of binary and multiple stars through radio astronomy techniques. There are about 85 binary pulsars and two multiple systems known. Currently there is no formal system of designating these companions.
\end{abstract}

Through the technique of Very Long Baseline Interferometry, radio astronomy provides the angular resolution to separate sources into different components. However, most stars are weak radio sources and few have been resolved (e.g. Ransom et al. 2003).

The discovery of pulsars allowed binary (or multiple) stellar systems to be resolved using the techniques of spectroscopy. Because of the fantastic precision of pulsars as clocks, pulsar spectroscopy is orders of magnitude more sensitive than even the most precise optical spectroscopy, allowing the detection of (for example) the first extra-Solar-System planetary system (Wolszczan \& Frail 1992). Of the $\sim 1500$ known pulsars, about 85 are members of binary or higher order systems. Most millisecond pulsars are binary. The companion star, usually a white dwarf, is rarely seen directly - in some cases the star has been identified through optical modulation at the binary period (e.g. Stappers et al. 2001) and two pulsars, PSR J0045-7319 and PSR B1259-63, have B-star companions identified through positional coincidence.

There is no current formal designation system for the companion in pulsar binary systems - they are simply referred to as "the companion of" the pulsar. Only two pulsars have evidence for more than one companion. The "planet pulsar", PSR B1257+12, has at least three planetary-mass companions, two with masses of about four Earth-masses and one with a mass close to that of the Moon (Wolszczan et al. 2000). These companions have been given letters, A, B and C in the order of their discovery, appended to the pulsar name. PSR B1620-26 is a binary pulsar with a white-dwarf companion in a 191-day orbit. Long-term pulse timing measurements indicate the presence of a second companion of mass $\sim 0.01 \mathrm{M}_{\odot}$ in an $\sim 100$-year orbit (Thorsett et al. 1999). Currently there is no separate designation for this companion.

In the future, with high-sensitivity radio telescopes such as the Square Kilometer Array, the number of known binary and multiple pulsar systems will certainly increase. It is important that pulsars be included in multiple-star and planetary-system catalogues and now is a good time to start. 


\section{References}

Ransom, R.R., Bartel, N., Bietenholz, M.F., Ratner, M.I., Lebach, D.E., Shapiro, I.I., \& Lestrade, J.-F. 2003, ApJ, 587, 390

Stappers, B.W., van Kerkwijk, M.H., Bell, J.F., \& Kulkarni, S.R. 2001, ApJ, 548, L183

Thorsett, S.E., Arzoumanian, Z., Camilo, F., \& Lyne, A.G. 1999, ApJ, 523, 763

Wolszczan, A. \& Frail, D.A. 1992, Nature, 355, 145

Wolszczan, A., Hoffman, I.M., Konacki, M., Anderson, S.B., \& Xilouris, K.M. 2000, ApJ, 540, L41

\section{Discussion}

DICKEL: Since so few binary systems are planets within pulsars this is a good opportunity to start entering them in the WMC and the pulsar community needs to label components and do it this way.

MANCHESTER: Fine, it would work as the need arises. This is not a problem at present. We just say "companion" of pulsar. 